\title{
New chronology of medieval objects in the Northern Black Sea region according to the method of determining calcite main peak intensity
}

\author{
Victoria Pishchulina* and Vladimir Kotlyar \\ Don State Technical University, 344002, Rostov-on-Don, Russia
}

\begin{abstract}
Determining the ancient architectural and cultural monuments' age is an important scientific problem. The article presents the results of the ancient brickwork lime mortars study. The portlandite transformation mechanism, which initially constitutes the basis of lime mortar, into calcite is shown. It has been established that this process takes from 100 to 200 years under natural conditions and the speed of this process is influenced by temperature, humidity, peculiarities of interaction with carbon dioxide contained in the air, etc. The examples showing that portlandite is completely transformed into calcite in masonry mortars of the 18th century, and that portlandite has not been found in older mortars are given. It was determined that after portlandite transition to calcite with increasing age, an increase in the calcitere crystallization degree is observed and this is manifested in a higher intensity of calcite peaks (especially the main peak $3.03 \AA$ ), increase in the crystallinity index - the width of the peak at half maximum (FWHM) or the main peak integral width, that is, the ratio of the area to the height of the peak above the background. Factual data, which show that in older lime solutions the degree of recrystallization of calcite is higher than in younger ones, are presented. This moment makes it possible to indirectly determine the relative age of brick and masonry of various monuments with architectural heritage, which is especially relevant for the South of Russia, where the objects have been preserved using lime mortars of the northern provinces in the Byzantine ecumene and other periods of various cultures.
\end{abstract}

\section{Discussion}

A. Grazzini, [1] applied the non-destructive methods to study the characteristics of historical stone and brickwork without compromising the artistic value of the monumental building. He used sound tests to characterize the stone walls at the Sanctuary of Santa Maria delle Grazie in Varoni, which revealed the texture and structural features of the masonry, and also confirmed the ineffectiveness of strengthening it with mortar injections.

\footnotetext{
* Corresponding author: viktvlad@mail.ru
} 
The problem of estimating the age of lime solutions affects not only historical and cultural aspects, but also archaeological ones, since organogenic limes tones with various calcite fossils were often used to obtain them. Falkenberg, J et al. [2] in his research presented evidence of the widespread use of organogenic limes tones for the lime solutions production and indirect signs of determining their age. Daugbjerg T. S. et al. [3] used radiocarbon dating techniques for historic stone structures containing organic remains. They presented sampling methods for radiocarbon dating of mortars. The authors showed that the samples of ancient mortars can contain various types of organic residues and, accordingly, carbon, which complicates correct testing and can lead to inconclusive results even when using modern methods.

Dating is especially important for specialists in the history of architecture, archaeologists, and historians. Knowing the absolute or even relative age of ancient building objects, specialists can draw many reliable conclusions in their fields of knowledge. Pishchulina V. et al. [4, 5] carried out a comprehensive research on the medieval lime mortars study using chemical, petrographic and X-ray phase analyzes. They investigated lime mortars of ancient buildings in the south of Russia, Armenia, Georgia, Greece, Turkey, Abkhazia and other regions. The results of the analyzes confirmed the estimated dates of the foundation of the second line, for example, of defense of the Anakopia fortress within 570-580 years, the reconstruction of the gate tower in 910-930 and the entrance gate - 950. Analysis of the lime mortars of the church near Anakopia (Akuakh temple) gave the construction time of 650-680 years. The method proposed by the authors for determining and clarifying the age of brick and masonry using lime mortars made it possible to revise the existing approaches to the dating of some cultural heritage sites.

Currently, in the history of architecture and archeology, there are many direct and indirect methods for determining the age of certain ancient building objects:

- historical and architectural methods: (Sanjurjo-Sánchez J [6], Batt C. [7]) calendar, typological, stratigraphic dating, serialization, etc.;

- physical and chemical methods (Mattinson, J [8], Thomsen, K.J. [9]): thermo-luminescent

method, electron paramagnetic resonance method, dating by remanent magnetization,

by racemization of amino acids, radiometric, potassium-argon dating, etc.

Aluker N. et al. in [10] investigated the possibility of using the thermo-luminescent method for dating fossilized paleontological remains of animals. The authors note a relatively simple application and a wide range of chronological periods during which the method gives reliable results with minimal errors. Using the thermo-luminescent method, the authors determined the different ages of the studied mammoth remains: from 12 to 100 thousand years. However, it is known that the thermo-luminescent method works well over long time intervals (Goedicke et al. [11]) and leads to large errors in small ones - for an age of up to 1000 years, the method leads to significant errors.

The radiocarbon method of dating organic remains by measuring the content of the radioactive isotope in the material is now widespread ${ }^{14} \mathrm{C}$ in relation to stable isotopes of carbon. Baydoun R. et al. [12] investigated the changes in atmospheric $\mathrm{CO}_{2}$ as a result of anthropogenic activities. We analyzed the samples of evergreen and deciduous treesleaves, as well as the seasonal leaves of small plants in the areas of industrial facilities with a $\mathrm{CO}_{2}$. The data showed that the concentration ${ }^{14} \mathrm{C}$ in the studied areas was significantly lower due to the release of anthropogenic $\mathrm{CO}_{2}$, than on clean territory.

Age-related petrographic analysis of mortar samples from Roman monuments including Portico Emilia, Temple of Concordia, Temple of Dioscuri, Temple B and other structures were carried out by Marra F. et al. [13]. The authors examined the volcanic rocks used in the mortars of the ancient Rome buildings from the beginning of the second century BC to the early imperial era in order to establish their pyroclastic origin (Pozzolane Rosse). The 
key issues in the study of mortars are usually their strength characteristics [14-16], which are determined, among other things, by non-destructive methods $[17,18]$.

In an article by Giaccone et al. [19] the effect of moisture on the specific gravity of masonry walls made of ceramic bricks and lime mortars of various monuments of architectural heritage was studied. The experimental studies of aging clutches have been carried out. It has been shown that moisture penetration causes an increase in masonry weight by more than $20 \%$. This indicator can be used in the general structural assessment of historic stone buildings. Determination of the characteristics and durability of mortars for their correct use and preservation of architectural monuments and historical heritage was carried out by Fernandez F. et al [20]. They studied the solutions based on lime metakaolin and hydraulic lime - metakaolin with the addition of nano- $\mathrm{TiO}_{2}$ and perlite. It has been shown that the solutions with pearlite and nano- $\mathrm{TiO}_{2}$ are the most effective, which makes them suitable for the preservation of monuments of cultural heritage.

Samples of mortars from Arslantepe (Turkey) provide unique information on the production and use of lime during the late Eneolithic period (4th millennium BC). A versatile approach to the study of lime mortars was carried out by Mignardi S. et al. [21], including polarized light microscopy (PLM), X-ray powder diffraction (XRPD) and scanning electron microscopy combined with energy dispersive spectroscopy (SEM-EDS), was used to characterize objects belonging to the Late Chalcolithic 3-4 (3800-3400 BC). Similar research methods based on X-ray fluorescence analysis, X-ray diffraction analysis and scanning electron microscopy of building solutions were carried out by Pavlík et al. [22]. Their compatibility and effectiveness have been shown for masonry and plastering materials for the historically valuable buildings' restoration.

The aim of the work carried out by the authors is to develop a method for the relative determination of the brick and masonry objects of cultural heritage age up to 2000 years, based on X-ray phase analysis according to the calcite recrystallization degree in lime solutions.

For the research, the samples of lime mortars were selected at various objects of the architectural heritage of the Northern Black Sea region and Crimea. In total, in the 2020 session, 149 samples. Objects for the research were classified into "reference", for which there are serious architectural studies, and they are dated by researchers, archaeologists and architects, and "controversial", for which there is no accurate and generally accepted dating data. We also studied the samples of natural carbonate rocks located near the sampling sites and from which lime was most likely obtained for solutions.

The results of the study and their correlation with the dating of archaeologists and architects, documentary sources showed that the main determining age of the elements is the intensity of the main peak of calcite, especially the main peak $3.03 \AA$ (in the sight 20 29.4).

Several samples of solutions were taken from each object in accordance with the periodization of parts of the building, aboveground and underground parts, and solar illumination. These factors were taken into account due to the fact that, as it is known, the rates of chemical reactions, and accordingly the transformation of portlandite into calcite and its recrystallization, largely depend on temperature and humidity. However, the studies have shown that this only affects the early stage of strengthening the solution - in the first 100 years.

To confirm the method, the degree of calcitere crystallization was also investigated, namely the value crystallinity index - the width of the peak at half maximum (FWHM) or the integral width of the main peak, that is, the area ratio to the height of the peak above the background, and it turned out that in older solutions these indicators are always $(100 \%)$ higher than in younger. 
The processes of portlandite carbonation $\left(\mathrm{Ca}(\mathrm{OH})_{2}\right)$, which depend on many technological factors: the dispersion of lime particles, the water content of the solution, temperature fluctuations, the concentration of carbon dioxide, as well as the presence of substances that contribute to an increase in the concentration $\mathrm{CO}_{2}$ inside the crystallizing mass were studied to verify the method. For example, by introducing organic materials: milk, blood, decoction of tree bark, etc., as practiced by ancient Russian masters, as well as the carbonization time. The last of the listed factors is decisive at the recrystallization stage, when the environment parameters are fairly uniform, and the change in the content of atmospheric $\mathrm{CO}_{2}$ and seasonal temperature fluctuations can be neglected on the scale of estimates at the decade or century level. Only long-term additional humidification is important, which promotes the dissociation of carbonates and bicarbonates with carbonic acid and the activation of ionexchange reactions in the liquid phase.

In parallel with carbonization, the solution can gain strength due to the interaction of calcium hydroxide with reactive types of silica, which is present in various rocks and ceramics - volcanic tuff and volcanic ash, siliceous opal-cristobalite rocks, volcanic acid rocks, ceramic battle and others. However, all this refers to the earlier stages of "hardening" of the solution. The last and longest stage of the "life" of a lime solution is the stage, conventionally called by us the stage of calcite recrystallization, accompanied by the growth of calcite microcrystals and an increase in the crystal lattice structural ordering degree. And at this stage, as studies have shown, the above-mentioned factors no longer matter.

Considering the above-said, we have developed a method for preparing the samples for X-ray studies, which consists in "soft" grinding of samples, since secondary calcite - the binder mass itself - is the least strong component of the solution, and the separation of a fine fraction $(0-50 \mu \mathrm{m})$ consisting of secondary calcite. For this, at the beginning, the existing coarse aggregate (fraction more than $5 \mathrm{~mm}$ ) was removed from the solution samples, after which the samples were ground with a rubber pestle in a porcelain mortar. This was done so that only the least strong binder mass was destroyed, and the existing fine aggregate, consisting mainly of quartz sand and other rocks, represented mainly with a fraction of more than $0.1 \mathrm{~mm}$, was not crushed. After grinding, the prepared mass was sieved on a sieve with a mesh size of $50 \mu \mathrm{m}$ for X-ray studies.

In the process of preparing samples and separating secondary calcite, we tried to achieve the minimum content of other minerals and rocks - quartz, sandstones, ferruginous and other minerals. If carbonate rocks were used as a solution filler, they tried to exclude the ingress of primary (natural) calcite into the sample or to achieve its minimum content. In any case, natural limestone has a significantly higher strength in comparison with a binder mass and a fractional composition of more than $0.1 \mathrm{~mm}$, therefore, it is not difficult to isolate it during sample preparation.

When interpreting and comparing the results obtained, we were guided by the confirmed historical data and reference the samples with a known age [30]. The studies were carried out on an ARLX'TRA X-ray diffractometer (Thermo Fisher Scientific, Waltham, MA USA) under the same shooting conditions. All samples prepared for X-ray studies were saved for the repeated studies, and some of the samples with a confirmed age were saved as reference standards (Table 1).

Table 1. List of objects investigated in 2020.

\begin{tabular}{|c|c|c|c|c|c|c|c|}
\hline No & $\begin{array}{c}\text { Registration } \\
\text { number }\end{array}$ & $\begin{array}{c}\text { Location, } \\
\text { object } \\
\text { name }\end{array}$ & $\begin{array}{c}\text { Sampling } \\
\text { location }\end{array}$ & $\begin{array}{c}\text { The calcite } \\
\text { crystallinity } \\
\text { main peak } \\
\text { intensity (by } \\
\text { instrument } \\
\text { ARLXTRA) }\end{array}$ & $\begin{array}{c}\text { Avera- } \\
\text { ge }\end{array}$ & $\begin{array}{c}\text { Dated by } \\
\text { the main } \\
\text { peak } \\
\text { intensity of } \\
\text { calcite } \\
\text { (ARLXTR } \\
\text { Adevice) }\end{array}$ & $\begin{array}{c}\text { Common } \\
\text { Dating, } \\
\text { century }\end{array}$ \\
& & & & & & & \\
& & & & & & & \\
\end{tabular}




\begin{tabular}{|c|c|c|c|c|c|c|c|}
\hline & & $\begin{array}{l}\text { Abkhazia } \\
2020\end{array}$ & & & & & \\
\hline & & Standard & & & & & \\
\hline 1. & EA-20-TS-1 & $\begin{array}{l}\text { Tsand- } \\
\text { ripsh }\end{array}$ & column & 5200 & 4800 & $5-6$ & 6 \\
\hline 2. & EA-20-TS-2 & Tsandripsh & porch & $\begin{array}{l}3050-2020 \\
3150-2019\end{array}$ & & 11 & 10 \\
\hline 3. & EA-20-TS-3 & Tsandripsh & altar & 5000 & & 6 & 6 \\
\hline 4. & EA-20-TS-4 & Tsandripsh & Wall south & 4200 & & 7 & 10 \\
\hline 5. & EA-20-TS-5 & Tsandripsh & Arch & 4700 & & 6 & 6 \\
\hline 6. & $\begin{array}{l}\text { EA-20- } \\
\text { MOK-1 }\end{array}$ & Mokvi & Masonry & 2640 & & 12 & 986 \\
\hline 7. & $\begin{array}{c}\text { EA-20-MOK- } \\
2\end{array}$ & Mokvi & $\begin{array}{l}\text { Wall } \\
\text { outside }\end{array}$ & 2390 & & 13 & 986 \\
\hline 8. & $\begin{array}{c}\text { EA-20-MOK- } \\
3\end{array}$ & Mokvi & $\begin{array}{l}\text { Masonry } \\
\text { from the } \\
\text { door }\end{array}$ & $\begin{array}{l}3500-2020 \\
3050-2019\end{array}$ & 3500 & End 10 & 986 \\
\hline 9. & $\begin{array}{c}\text { EA-20-MOK- } \\
4\end{array}$ & Mokvi & $\begin{array}{l}\text { Sea mold } \\
\text { temple }\end{array}$ & 5200 & & $\begin{array}{c}\text { Beginning } \\
6\end{array}$ & 986 \\
\hline 10. & $\begin{array}{c}\text { EA-20-MOK- } \\
5\end{array}$ & Mokvi & Outside & 2350 & & 13 & 986 \\
\hline 11. & $\begin{array}{c}\text { EA-20-MOK- } \\
9\end{array}$ & Mokvi & At the door & 2880 & & 12 & 986 \\
\hline 12. & $\begin{array}{c}\text { EA-20-Khas- } \\
1\end{array}$ & Khashupse & $\begin{array}{l}\text { Upper } \\
\text { tower }\end{array}$ & 4720 & & 6 & 6 \\
\hline 13. & $\begin{array}{c}\text { EA-20-Khas- } \\
3\end{array}$ & Khashupse & $\begin{array}{l}\text { Temple } \\
\text { altar }\end{array}$ & 5450 & 4800 & $\begin{array}{c}\text { Beginning } \\
6\end{array}$ & 6 \\
\hline 14. & $\begin{array}{c}\text { EA-20-Khas- } \\
4\end{array}$ & Khashupse & $\operatorname{tank}$ & 4240 & & 7 & 6 \\
\hline 15. & $\begin{array}{l}\text { EA-20- } \\
\text { AbAn-1 }\end{array}$ & AbaAnta & 1 wal lwest & 4500 & & 8 & 8 \\
\hline 16. & $\begin{array}{l}\text { EA-20- } \\
\text { AbAn-2 }\end{array}$ & AbaAnta & 2 wall east & 4200 & 3900 & 8 & 8 \\
\hline 17. & $\begin{array}{l}\text { EA-20- } \\
\text { AbAn-3 }\end{array}$ & AbaAnta & 3 porch & 3600 & & 10 & 8 \\
\hline 18. & $\begin{array}{l}\text { EA-20- } \\
\text { AbAn-4 }\end{array}$ & AbaAnta & 4 lining & 3300 & & 10 & 8 \\
\hline 19. & $\begin{array}{c}\text { EA-20-MU- } \\
12\end{array}$ & Mussera & $\begin{array}{l}\text { Building } \\
\text { nearby }\end{array}$ & 4950 & & $5-6$ & - \\
\hline 20. & EA-20-MU-4 & Mussera & $\begin{array}{l}\text { North } \\
\text { ernporch }\end{array}$ & 4900 & 4900 & $5-6$ & $6-7$ \\
\hline 21. & $\begin{array}{c}\text { EA-20-MU- } \\
10\end{array}$ & Mussera & $\begin{array}{l}\text { Main } \\
\text { facade }\end{array}$ & $\begin{array}{l}4900-2020 \\
4750-2019\end{array}$ & & 6 & 10 \\
\hline 22. & EA-20-MU-3 & Mussera & North wall & 4000 & & 7 & $6-7$ \\
\hline 23. & EA-20-MU-8 & Mussera & $\begin{array}{l}\text { Altar of the } \\
\text { northern } \\
\text { apse. }\end{array}$ & 4300 & & 7 & - \\
\hline 24. & $\begin{array}{c}\text { EA-20-MU- } \\
11\end{array}$ & Mussera & Galery & 4100 & & 7 & - \\
\hline 25. & EA-20-MU-7 & Mussera & $\begin{array}{l}\text { Choir } \\
\text { coating }\end{array}$ & 3500 & & 10 & - \\
\hline 26. & EA-20-MU-9 & Mussera & $\begin{array}{l}\text { Altar } \\
\text { outside }\end{array}$ & 2500 & & 12 & - \\
\hline 27. & $\begin{array}{c}\text { EA-20-MU- } \\
13\end{array}$ & Mussera & $\begin{array}{l}\text { Western } \\
\text { wall with } \\
\text { choirs }\end{array}$ & 3200 & 3300 & 10 & 10 \\
\hline 28. & EA-20-MU-5 & Mussera & $\begin{array}{l}\text { Altar } \\
\text { outside }\end{array}$ & 2500 & & 12 & - \\
\hline 29. & $\begin{array}{c}\text { EA-20- } \\
\text { BAGR-1 }\end{array}$ & $\begin{array}{l}\text { Bagrat's } \\
\text { Castle }\end{array}$ & gates & 4400 & & 6 & 11 \\
\hline 30. & EA-20- & Bagrat's & $1^{\text {st }}$ stage & 3800 & & 9 & 11 \\
\hline
\end{tabular}




\begin{tabular}{|c|c|c|c|c|c|c|c|}
\hline & BAGR-2 & Castle & & & & & \\
\hline 31. & $\begin{array}{l}\text { EA-20- } \\
\text { BAGR-3 }\end{array}$ & $\begin{array}{l}\text { Bagrat's } \\
\text { Castle }\end{array}$ & $2^{\text {nd }}$ stage & 2800 & & 11 & 13 \\
\hline 32. & $\begin{array}{c}\text { EA-20- } \\
\text { LYKHC-1 }\end{array}$ & $\begin{array}{l}\text { Lykhny's } \\
\text { Castle }\end{array}$ & Upper tier & 3400 & & 10 & 11 \\
\hline 33. & $\begin{array}{c}\text { EA-20- } \\
\text { LYKHC-2 }\end{array}$ & $\begin{array}{l}\text { Lykhny's } \\
\text { Castle }\end{array}$ & vault & 4500 & & 6 & 11 \\
\hline 34. & $\begin{array}{c}\text { EA-20- } \\
\text { LYKHC-3 }\end{array}$ & $\begin{array}{l}\text { Lykhny's } \\
\text { Castle }\end{array}$ & Lower tier & 3800 & & 8 & 9 \\
\hline 35. & $\begin{array}{c}\text { EA-20- } \\
\text { LYKHC-4 }\end{array}$ & $\begin{array}{l}\text { Lykhny's } \\
\text { Castle }\end{array}$ & gate & 3400 & & 10 & 9 \\
\hline 36. & $\begin{array}{l}\text { EA-20- } \\
\text { LYKH-1 }\end{array}$ & $\begin{array}{l}\text { Lykhny } \\
\text { Church }\end{array}$ & $\begin{array}{l}\text { building } \\
\text { extension } \\
\text { was } \\
\text { destroyed }\end{array}$ & 2600 & & 12 & 9 \\
\hline 37. & $\begin{array}{c}\text { EA-20- } \\
\text { LYKH-2 }\end{array}$ & $\begin{array}{l}\text { Lykhny } \\
\text { Church }\end{array}$ & $\begin{array}{l}\text { Side chapel } \\
\text { south }\end{array}$ & 4000 & 3800 & 9 & 11 \\
\hline 38. & $\begin{array}{l}\text { EA-20- } \\
\text { LYKH-3 }\end{array}$ & $\begin{array}{l}\text { Lykhny } \\
\text { Church }\end{array}$ & $\begin{array}{l}\text { Coating } \\
\text { inside the } \\
\text { columns }\end{array}$ & 3600 & & 10 & 11 \\
\hline 39. & $\begin{array}{c}\text { EA-20- } \\
\text { LYKH-5 }\end{array}$ & $\begin{array}{l}\text { Lykhny } \\
\text { Church }\end{array}$ & column & 4100 & & 9 & 11 \\
\hline 40. & E-20 BZ-1 & Bzyb & $\begin{array}{l}\text { Tower in } \\
\text { front of the } \\
\text { temple }\end{array}$ & $\begin{array}{l}4150-2020 \\
4000-2019\end{array}$ & & 6 & 10 \\
\hline 41. & E-20 BZ-2 & Bzyb & $\begin{array}{l}\text { Tower } \\
\text { below }\end{array}$ & 4000 & & 7 & 11 \\
\hline 42. & E-20 BZ-3 & Bzyb & Old temple & $\begin{array}{l}4600-2020 \\
4600-2019 \\
\end{array}$ & & $\begin{array}{c}\text { Beginning } \\
6\end{array}$ & 6 \\
\hline 43. & E-20 BZ-4 & Bzyb & church & $\begin{array}{l}3600-2020 \\
3300-2019\end{array}$ & & End 9-10 & 10 \\
\hline 44. & E-20 BZ-5 & Bzyb & Main gate & 3350 & & 10 & 8 \\
\hline 45. & E-20 BZ-7 & Bzyb & $\begin{array}{l}\text { The wall at } \\
\text { the gate }\end{array}$ & 3200 & & 10 & 8 \\
\hline 46. & E-20 SC-1 & $\begin{array}{l}\text { Simon the } \\
\text { Canaanite }\end{array}$ & $\begin{array}{l}\text { Altar } \\
\text { outside }\end{array}$ & 3100 & & End 10 & 10 \\
\hline 47. & E-20 SC-2 & $\begin{array}{l}\text { Simon the } \\
\text { Canaanite }\end{array}$ & $\begin{array}{l}\text { Cladding } \\
\text { outside }\end{array}$ & 1200 & & 18 & 10 \\
\hline 48. & E-20-AN-1 & Anacopia & $\begin{array}{l}\text { Temple at } \\
\text { turn } 3 \text { aps }\end{array}$ & 5350 & & $\begin{array}{c}\text { Beginning } \\
6 \\
\end{array}$ & 8 \\
\hline 49. & E-20-AN-2 & Anacopia & $\begin{array}{l}\text { The gate at } \\
\text { the old } \\
\text { tower }\end{array}$ & 4770 & & $\begin{array}{c}\text { Beginning } \\
6\end{array}$ & 6 \\
\hline 50. & E-20-AN-3 & Anacopia & $\begin{array}{ll}\text { Wall near } \\
\text { the } \\
\text { tower }\end{array}$ & 5500 & & $\begin{array}{c}\text { Beginning } \\
6\end{array}$ & 10 \\
\hline 51. & E-20-AN-4 & Anacopia & $\begin{array}{l}\text { Gate tower } \\
\text { 6th century }\end{array}$ & 5025 & & $\begin{array}{c}\text { Beginning } \\
6\end{array}$ & 6 \\
\hline 52. & E-20-AN-5 & Anacopia & $\begin{array}{l}\text { Temple at } \\
\text { turn } 1\end{array}$ & 4120 & & 7 & 7 \\
\hline 53. & E-20-AN-6 & Anacopia & $\begin{array}{l}\text { The temple } \\
\text { is on the } \\
\text { territory } \\
\text { citadel } \\
\text { down }\end{array}$ & 4770 & & $\begin{array}{c}\text { Beginning } \\
6\end{array}$ & 10 \\
\hline 54. & E-20-AN-7 & Anacopia & citadel & $\begin{array}{l}4900-2020 \\
4600-2019\end{array}$ & & $\begin{array}{c}\text { Beginning } \\
6\end{array}$ & 6 \\
\hline 55. & E-20-AN-8 & Anacopia & Tower wall & 4270 & & End 6 & 6 \\
\hline 56. & E-20-AN-9 & Anacopia & $\begin{array}{l}\text { Tower } 2 \\
\text { bottom, } \\
\text { lining }\end{array}$ & 4460 & & 6 & 6 \\
\hline 57. & E-20-AN-12 & Anacopia & Out & 3700 & & 10 & 10 \\
\hline
\end{tabular}




\begin{tabular}{|c|c|c|c|c|c|c|c|}
\hline & & & $\begin{array}{l}\text { building } \\
\text { with gate } \\
\text { figs }\end{array}$ & & & & \\
\hline 58. & E-20-AN-13 & Anacopia & $\begin{array}{l}\text { Temple } \\
\text { chapel }\end{array}$ & 5300 & & $\begin{array}{c}\text { Beginning } \\
6 \\
\end{array}$ & 10 \\
\hline 59. & E-20-AN-17 & Anacopia & $\begin{array}{l}\text { Temple top } \\
\text { part }\end{array}$ & 5250 & & $\begin{array}{c}\text { Beginning } \\
6 \\
\end{array}$ & 10 \\
\hline 60. & E-20-AN-18 & Anacopia & Wall 2 lines & 4000 & & 6 & 6 \\
\hline 61. & E-20-AN-19 & Anacopia & cistern & 5650 & & $\begin{array}{c}\text { Beginning } \\
6\end{array}$ & 4 \\
\hline 62. & E-20-AN-20 & Anacopia & $\begin{array}{l}\text { Wall with } \\
\text { Alkhas }\end{array}$ & 5330 & & $\begin{array}{c}\text { Beginning } \\
6\end{array}$ & 6 \\
\hline 63. & E-20-AN-21 & Anacopia & East tower & 4650 & & 6 & 11 \\
\hline 64. & E-20-AN-22 & Anacopia & $\begin{array}{l}\text { from the } \\
\text { East }\end{array}$ & 5150 & & $\begin{array}{c}\text { Beginning } \\
6\end{array}$ & 19 \\
\hline 65. & E-20-AN-23 & Anacopia & $\begin{array}{l}\text { Temple top } \\
\text { vault }\end{array}$ & 3600 & & 10 & 9 \\
\hline 66. & E-20-AN-24 & Anacopia & $\begin{array}{l}\text { Temple } \\
\text { upper } \\
\text { vestibule }\end{array}$ & 4400 & & 6 & 6 \\
\hline 67. & E-20-AN-25 & Anacopia & $\begin{array}{l}\text { Temple } \\
\text { upper altar }\end{array}$ & 4880 & & $\begin{array}{c}\text { Beginning } \\
6\end{array}$ & 6 \\
\hline 68. & E-20-AN-10 & Anacopia & $\begin{array}{l}\text { Gate tower } \\
\text { bottom }\end{array}$ & 4970 & & $\begin{array}{c}\text { Beginning } \\
6 \\
\end{array}$ & 6 \\
\hline 69. & E-20-BAM-1 & Bambora & $\begin{array}{l}\text { Temple } \\
\text { altar }\end{array}$ & $\begin{array}{l}3500-2020 \\
3700-2019 \\
\end{array}$ & & End 9 & 9 \\
\hline 70. & E-20-BAM-2 & Bambora & $\begin{array}{l}\text { Temple } \\
\text { extreme }\end{array}$ & 2550 & & 12 & 6 \\
\hline 71. & E-20-BAM-2 & Bambora & $\begin{array}{l}\text { Temple } \\
\text { average }\end{array}$ & 3500 & & $\begin{array}{c}\text { Beginning } \\
10\end{array}$ & 7 \\
\hline & & CRIMEA & & & & & \\
\hline 72. & $\begin{array}{c}\text { E-20-CR- } \\
\text { TIR-1 }\end{array}$ & Tiritaka & $\begin{array}{ll}\begin{array}{l}\text { House } \\
\text { plaster }\end{array} & \text { of } \\
\end{array}$ & 2100 & & 17 & 3 \\
\hline 73. & $\begin{array}{l}\text { E-20-CR- } \\
\text { TIR-2 }\end{array}$ & Tiritaka & winery & 1530 & & 18 & 3 \\
\hline 74. & $\begin{array}{l}\text { E-20-CR- } \\
\text { TIR-3 }\end{array}$ & Tiritaka & house & 2700 & & 15 & 3 \\
\hline 75. & $\begin{array}{l}\text { E-20-CR- } \\
\text { TIR-4 }\end{array}$ & Tiritaka & $\begin{array}{l}\text { Temple } \\
\text { 3cen. }\end{array}$ & 2850 & & 15 & 3 \\
\hline 76. & $\begin{array}{l}\text { E-20-CR- } \\
\text { TIR-5 }\end{array}$ & Tiritaka & $\begin{array}{l}\begin{array}{l}\text { House } 3 \\
\text { cen. }\end{array} \\
\end{array}$ & 3000 & & 14 & 3 \\
\hline 77. & $\begin{array}{c}\text { E-20-CR- } \\
\text { TIR-6 } \\
\end{array}$ & Tiritaka & $\begin{array}{l}\text { Nymphaeu } \\
\mathrm{m}\end{array}$ & 4630 & & 12 & 4 \\
\hline 78. & $\begin{array}{l}\text { E-20-CR- } \\
\text { CHUF-1 }\end{array}$ & $\begin{array}{l}\text { Chufut- } \\
\text { Kale }\end{array}$ & Old wall & 4500 & & 12 & 6 \\
\hline 79. & $\begin{array}{l}\text { E-20-CR- } \\
\text { CHUF-2 }\end{array}$ & Chufut-kale & Gate & 4270 & & 12 & 6 \\
\hline 80. & $\begin{array}{l}\text { E-20-CR- } \\
\text { CHUF-3 }\end{array}$ & Chufut-kale & tower & 3250 & & 14 & 12 \\
\hline 81. & $\begin{array}{l}\text { E-20-CR- } \\
\text { CHUF-4 }\end{array}$ & Chufut-kale & tomb & 3800 & 3300 & 14 & 1380 \\
\hline 82. & $\begin{array}{l}\text { E-20-CR- } \\
\text { CHUF-5 }\end{array}$ & Chufut-kale & mosque & 3500 & & 14 & 1347 \\
\hline 83. & $\begin{array}{c}\text { E-20-CR-AL- } \\
3\end{array}$ & Alushta & $\begin{array}{l}\text { Second } \\
\text { Tower }\end{array}$ & 4300 & & 12 & 15 \\
\hline 84. & $\begin{array}{c}\text { E-20-CR-AL- } \\
5 \\
\end{array}$ & Alushta & 2 barbican & 4100 & & 12 & 15 \\
\hline 85. & $\begin{array}{l}\text { E-20-CR- } \\
\text { CHER-1 }\end{array}$ & $\begin{array}{l}\text { Cherso- } \\
\text { nesus }\end{array}$ & $\begin{array}{l}\text { Church No. } \\
9\end{array}$ & 4550 & & 9 & 6 \\
\hline 86. & $\begin{array}{l}\text { E-20-CR- } \\
\text { CHER-2 }\end{array}$ & $\begin{array}{l}\text { Cherso- } \\
\text { nesus }\end{array}$ & $\begin{array}{l}\text { Basilica on } \\
\text { the cliff }\end{array}$ & 4470 & & 9 & 6 \\
\hline 87. & E-20-CR- & Cherso- & Winemaker' & 3370 & & 15 & 4 \\
\hline
\end{tabular}




\begin{tabular}{|c|c|c|c|c|c|c|c|}
\hline & CHER-3 & nesus & $\begin{array}{l}\mathrm{s} \text { house } \\
\text { plastering }\end{array}$ & & & & \\
\hline 88. & $\begin{array}{l}\text { E-20-CR- } \\
\text { CHER-4 }\end{array}$ & $\begin{array}{l}\text { Cherso- } \\
\text { nesus }\end{array}$ & House & 2650 & & 16 & 10 \\
\hline 89. & $\begin{array}{l}\text { E-20-CR- } \\
\text { CHER-5 }\end{array}$ & $\begin{array}{l}\text { Cherso- } \\
\text { nesus }\end{array}$ & $\begin{array}{l}\text { Wine } \\
\text { maker's } \\
\text { house, with } \\
\text { bricks }\end{array}$ & 3200 & & 15 & 4 \\
\hline 90. & $\begin{array}{c}\text { E-20-CR- } \\
\text { BO-1 }\end{array}$ & Bogatoye & vault & 3850 & & 12 & 13 \\
\hline 91. & $\begin{array}{c}\text { E-20-CR-BO- } \\
2\end{array}$ & Bogatoye & $\begin{array}{l}\text { Western } \\
\text { wall }\end{array}$ & 2900 & 3700 & 12 & 13 \\
\hline 92. & $\begin{array}{c}\text { E-20-CR-BO- } \\
3\end{array}$ & Bogatoye & $\begin{array}{l}\text { Native } \\
\text { masonry } \\
\text { wall }\end{array}$ & 3700 & & 12 & 13 \\
\hline 93. & $\begin{array}{c}\text { E-20-CR-BO- } \\
4\end{array}$ & Bogatoye & $\begin{array}{l}\text { Outer } \\
\text { lining }\end{array}$ & 3950 & & 12 & 13 \\
\hline 94. & $\begin{array}{c}\text { E-20-CR-BO- } \\
6\end{array}$ & Bogatoye & roof & 3600 & & 13 & 13 \\
\hline 95. & $\begin{array}{c}\text { E-20-CR-BO- } \\
7\end{array}$ & Bogatoye & altar & 4000 & & 12 & 13 \\
\hline 96. & $\begin{array}{c}\text { E-20-CR-SU- } \\
1\end{array}$ & Sudak & $\begin{array}{l}\text { Central } \\
\text { Hall }\end{array}$ & 4350 & & 11 & 9 \\
\hline 97. & $\begin{array}{c}\text { E-20-CR-SU- } \\
2\end{array}$ & Sudak & Old temple & 4720 & & 10 & 9 \\
\hline 98. & $\begin{array}{c}\text { E-20-CR-SU- } \\
3\end{array}$ & Sudak & North wall & 4120 & 4800 & 11 & 9 \\
\hline 99. & $\begin{array}{c}\text { E-20-CR-SU- } \\
4\end{array}$ & Sudak & solution & 4350 & & 11 & 9 \\
\hline 100. & $\begin{array}{c}\text { E-20-CR-SU- } \\
5\end{array}$ & Sudak & $\begin{array}{l}\text { From } \\
\text { niche }\end{array}$ & 4600 & & 10 & 9 \\
\hline 101. & $\begin{array}{c}\text { E-20-CR-SU- } \\
6\end{array}$ & Sudak & $\begin{array}{l}\text { From the } \\
\text { altar }\end{array}$ & 5050 & & 10 & 9 \\
\hline 102. & $\begin{array}{c}\text { E-20-CR-SU- } \\
8\end{array}$ & Sudak & $\begin{array}{l}\text { From the } \\
\text { west wall }\end{array}$ & 5250 & & 10 & 9 \\
\hline 103. & $\begin{array}{l}\text { E-20-CR- } \\
\text { MP-1 }\end{array}$ & Morskoye & $\begin{array}{l}\text { From the } \\
\text { west wall }\end{array}$ & 4220 & & 11 & 15 \\
\hline 104. & $\begin{array}{c}\text { E-20-CR-AP- } \\
1\end{array}$ & $\begin{array}{ll}\text { Sudak } & 12 \\
\text { apostles } & \end{array}$ & bottom & 1950 & & 15 & 12 \\
\hline 105. & $\begin{array}{c}\text { E-20-CR-AP- } \\
2\end{array}$ & $\begin{array}{ll}\text { Sudak } & 12 \\
\text { apostles }\end{array}$ & top & 2900 & & 12 & 13 \\
\hline 106. & $\begin{array}{c}\text { E-20-CR- } \\
\text { ПАР-1 }\end{array}$ & $\begin{array}{l}\text { PartenitBa } \\
\text { silica }\end{array}$ & Old part & 4150 & & 11 & 8 \\
\hline 107. & $\begin{array}{c}\text { E-20-CR-oc- } \\
1\end{array}$ & $\begin{array}{l}\text { Old } \\
\text { CrimeaSur } \\
\text { pKhach }\end{array}$ & walls & 3400 & & 14 & 13 \\
\hline 108. & E-20-CR-oc-2 & $\begin{array}{l}\text { Old } \\
\text { CrimeaSurp } \\
\text { Khach }\end{array}$ & facing & 4800 & & 10 & 13 \\
\hline & & SOCHI & & & & & \\
\hline 109. & C-20-KN-1 & $\begin{array}{l}\text { Krion- } \\
\text { Neron }\end{array}$ & $\begin{array}{l}\text { Stone with } \\
\text { mortar }\end{array}$ & 3650 & & 10 & 12 \\
\hline 110. & C-20-KN-2 & $\begin{array}{l}\text { Krion- } \\
\text { Neron }\end{array}$ & 1 & 3500 & & 10 & 12 \\
\hline 111. & C-20-KN-3 & $\begin{array}{l}\text { Krion- } \\
\text { Neron }\end{array}$ & 3 & 3100 & & 11 & 12 \\
\hline 112. & C-20-KN-5 & $\begin{array}{l}\text { Krion- } \\
\text { Neron }\end{array}$ & $\begin{array}{l}\text { Knocked } \\
\text { out of the } \\
\text { ground }\end{array}$ & 4380 & & 8 & 12 \\
\hline 113. & C-20-LO-1 & LOO & $\begin{array}{l}\text { South } \\
\text { portico }\end{array}$ & 4070 & & 8 & 11 \\
\hline 114. & C-20-LO-2 & LOO & $\begin{array}{l}\text { South } \\
\text { portico }\end{array}$ & 4070 & & 7 & $10-11$ \\
\hline
\end{tabular}




\begin{tabular}{|c|c|c|c|c|c|c|}
\hline 115. & C-20-LO-3 & $\mathrm{LOO}$ & $\begin{array}{l}\text { From a } \\
\text { fallen piece } \\
\text { at the } \\
\text { entrance }\end{array}$ & 3570 & 10 & 11 \\
\hline 116. & C-20-LO-4 & $\mathrm{LOO}$ & column & 3100 & 11 & 11 \\
\hline 117. & C-20-LO-5 & $\mathrm{LOO}$ & Altar & 4050 & 7 & 11 \\
\hline 118. & C-20-KHO-1 & Khosta & $\begin{array}{l}\text { Tower No. } \\
1 \text { and the } \\
\text { wall }\end{array}$ & 2550 & 12 & 11 \\
\hline 119. & C-20-KHO-3 & Khosta & $\begin{array}{l}\begin{array}{l}\text { Tower } 2-2 \\
\text { wall }\end{array} \\
\end{array}$ & 3800 & 10 & 14 \\
\hline 120. & C-20-KHO-4 & Khosta & Tower 4 & 4300 & 8 & 15 \\
\hline 121. & C-20-KHO-5 & Khosta & Tower 5 & 4300 & 8 & 15 \\
\hline 122. & C-20-ACH-1 & Achipse & $\begin{array}{l}\text { Center } \\
\text { tower }\end{array}$ & 3700 & 10 & 6 \\
\hline 123. & C-20-ACH-2 & Achipse & $\begin{array}{l}\begin{array}{l}\text { Center } \\
\text { entrance }\end{array} \\
\end{array}$ & 4150 & 9 & 6 \\
\hline 124. & C-20-ACH-3 & Achipse & $\begin{array}{l}\text { Tower with } \\
\text { road } \\
\text { entrance }\end{array}$ & 3350 & 10 & 6 \\
\hline 125. & C-20-ACH-4 & Achipse & $\begin{array}{l}\text { Old } \\
\text { entrance }\end{array}$ & 3650 & 10 & 6 \\
\hline 126. & С-20-МА-1 & Mamayka & old & 3650 & 10 & 5 \\
\hline 127. & C-20-MA-2 & Mamayka & lining & 3750 & 10 & 5 \\
\hline 128. & C-20-MA-3 & Mamayka & 2 stage & 3200 & 10 & 5 \\
\hline 129. & C-20-MA-4 & Mamayka & - & 4300 & 6 & 5 \\
\hline 130. & C-20-LB1-1 & $\begin{array}{l}\text { Lesnianska } \\
\text { Basilica } 1 \\
\end{array}$ & tower & 4450 & 6 & 6 \\
\hline 131. & C-20-LB1-2 & $\begin{array}{l}\text { Lesnianska } \\
\text { Basilica } 1 \\
\end{array}$ & North Apse & 4350 & 6 & 6 \\
\hline 132. & C-20-LB1-3 & $\begin{array}{l}\text { Lesnianska } \\
\text { Basilica } 1 \\
\end{array}$ & In the altar & 3800 & 7 & 6 \\
\hline 133. & C-20-LB1-4 & $\begin{array}{l}\text { Lesnianska } \\
\text { Basilica } 1\end{array}$ & lining & 3200 & 10 & 6 \\
\hline 134. & $\begin{array}{c}\text { C-20-AKHS- } \\
1 \\
\end{array}$ & Akhstyr & altar & 3850 & 9 & 13 \\
\hline 135. & $\begin{array}{c}\text { C-20-AKHS- } \\
2 \\
\end{array}$ & Akhstyr & porch & 4200 & 7 & 14 \\
\hline 136. & $\begin{array}{c}\text { C-20-AKHS- } \\
3 \\
\end{array}$ & Akhstyr & $\begin{array}{l}\text { Indented } \\
\text { solution }\end{array}$ & 3700 & 10 & 14 \\
\hline 137. & $\begin{array}{c}\text { C-20-AKHS- } \\
4 \\
\end{array}$ & Akhstyr & $\begin{array}{l}\text { In the } \\
\text { ground }\end{array}$ & 2300 & 13 & 15 \\
\hline 138. & $\begin{array}{c}\text { C-20-MonD- } \\
1\end{array}$ & $\begin{array}{l}\text { Dragon's } \\
\text { mouth } \\
\text { monastery }\end{array}$ & tower & 3950 & 8 & 11 \\
\hline 139. & $\begin{array}{c}\text { C-20-MonD- } \\
2\end{array}$ & $\begin{array}{l}\text { Dragon's } \\
\text { mouth } \\
\text { monastery } \\
\end{array}$ & $\begin{array}{l}\text { Tower } \\
\text { inside }\end{array}$ & 4100 & 9 & 11 \\
\hline 140. & $\begin{array}{c}\text { C-20-MonD- } \\
3\end{array}$ & $\begin{array}{l}\text { Dragon's } \\
\text { mouth } \\
\text { monastery }\end{array}$ & $\begin{array}{l}\text { Indented } \\
\text { temple } \\
\text { entrance }\end{array}$ & 3500 & 10 & 7 \\
\hline 141. & $\begin{array}{c}\text { C-20-MonD- } \\
4\end{array}$ & $\begin{array}{l}\text { Dragon's } \\
\text { mouth } \\
\text { monastery }\end{array}$ & 1 wall & 4050 & 7 & 7 \\
\hline 142. & $\begin{array}{c}\text { C-20-MonD- } \\
5\end{array}$ & $\begin{array}{l}\text { Dragon's } \\
\text { mouth } \\
\text { monastery }\end{array}$ & 2 wall & 4050 & 7 & 11 \\
\hline 143. & $\begin{array}{c}\text { C-20-MonD- } \\
6\end{array}$ & $\begin{array}{l}\text { Dragon's } \\
\text { mouth } \\
\text { monastery }\end{array}$ & church & 4400 & 6 & 7 \\
\hline & \multicolumn{2}{|c|}{ Mountain part - standards } & & & & \\
\hline 144. & & Sentin & wall & 3350 & 10 & 10 \\
\hline
\end{tabular}




\begin{tabular}{|c|c|c|c|c|c|c|}
\hline & & church & & & & \\
\hline 145. & & $\begin{array}{l}\text { Sentin } \\
\text { church }\end{array}$ & $\begin{array}{l}\text { Support } \\
\text { under- } \\
\text { ground }\end{array}$ & 3400 & 10 & 10 \\
\hline & \multicolumn{6}{|c|}{ Standards of the last session Abkhazia } \\
\hline 146. & A-2 & Anacopia & $\begin{array}{l}\text { Gate } \\
\text { towerwall }\end{array}$ & $\begin{array}{l}5100-2020 \\
4400-2019\end{array}$ & Beginning6 & 6 \\
\hline 147. & A-5 & Anacopia & $\begin{array}{l}\text { Temple in } \\
\text { citadel }\end{array}$ & $\begin{array}{l}4700-2020 \\
4000-2019\end{array}$ & $\begin{array}{c}\text { Beginning } \\
6\end{array}$ & 6 \\
\hline 148. & A-8 & Gerzeul & Tower & $\begin{array}{l}3950-2020 \\
3800-2019 \\
\end{array}$ & $6-7$ & 6 \\
\hline 149. & A-45 & $\begin{array}{l}\text { Round } \\
\text { Tower , / } \\
\text { Anacopia }\end{array}$ & Gate arch & $\begin{array}{l}4700-2020 \\
4400-2019\end{array}$ & $\begin{array}{c}\text { Beginning } \\
6\end{array}$ & 10 \\
\hline
\end{tabular}

\section{Results}

As a result of the studies carried out, it was found that the presence of portlandite was not detected in the samples of lime mortars from the buildings of the 18th century and older $\mathrm{Ca}(\mathrm{OH})_{2}$, which manifests itself in the diffraction patterns by peaks corresponding to 2.63; $4.93 ; 1.93 \AA$. This is due to its complete transition to calcite. Newly formed calcite has a non-uniform crystalline structure with a predominance of microporous crystal aggregates and their intergrowths less than 5 microns in size. The morphology of calcite crystals is characteristic of this mineral. The dependence of the calcite crystals size on age has not been established.

The degree of calcitere crystallization and its maximum - the main peak $3.03 \AA$ better defined with slow motion radiographs in the sight area 20 29.4. Numerous analyzes made it possible to establish a clear relationship - the higher the age of the object, and, accordingly, the brick or masonry, confirmed by the architectural and archaeological data, the higher the calcite recrystallization degree formed from portlandite. It was also found that the growth of calcitere crystallization depends on many conditions and presumably in the first centuries the process is more intensive, and with increasing age the rate slows down.

The study of ancient lime mortars by the proposed method allows in some cases to confirm, clarify, and in some cases to establish the age of monuments of architectural heritage and their individual parts. However, to develop a full-fledged methodology, taking into account the complexity and versatility of the tasks, it is necessary to accumulate actual data, select the reference samples for different regions and, most importantly, the coordinated work of various specialists.

\section{Conclusion}

Preliminary comparisons made by us showed that for most of the objects (80-85\%) the relative age, determined by the calcite recrystallization degree, correlates with the historical age of the objects of the architectural and cultural heritage, derived on the basis of architectural, historical and archaeological research, and for some objects - no. These are mainly objects with controversial dating. However, for such objects, the dating of lime mortars obtained by the authors of the article coincided with the opinion of a number of the researchers on the construction periods of these objects.

Our proposed method for determining and clarifying the age of objects of architectural heritage by the calcite recrystallization degree of ancient lime solutions makes it possible to supplement and clarify the data of historiography and architectural studies by the age of architectural monuments, since most of the monuments lack construction inscriptions and 
evidence from written sources. For individual objects, it was possible to determine the historical stages of construction. The data obtained on a number of objects radically change the generally accepted dating and require further clarification.

The phenomenon of portlandite trans formation into calcite and subsequent recrystallization of calcite can be used to estimate the age of ancient limestone masonry for a local group of objects. Diffraction studies of powder samples show a change in the parameters of the main reflection of calcite (hkl 104), which is determined by the increase in the calcite crystallinity over time. In the presence of a number of samples with a known historical age for a group of objects, the tendency between the parameters of the considered diffraction maximum and the building age is estimated, which makes it possible to use this dependence to determine the age of the objects under study. The reliability of the results obtained is largely determined by the analytical samples' preparation quality.

This research was funded by Russian Foundation for Fundamental Research, grant number 19-012$00402 \backslash 21$.

\section{References}

1. A. Grazzini, Appl. Sci.9, 5148 (2019). https://doi.org/10.3390/app9235148

2. J. Falkenberg, J. Mutterlose, U. Kaplan, Archaeometry 63, 1, 19-39 (2021). https://doi.org/10.1111/arcm.12626

3. T.S. Daugbjerg, A. Lindroos, J. Heinemeier, Å. Ringbom, G. Barrett, D. Michalska, I. Hajdas, R. Raja, J. Olsen, Archaeometry, 2021. https://doi.org/10.1111/arcm.12648

4. V. Pishchulina, V. Kotlyar, A. Argun, Integrated Cross-disciplinary Approach to Dating the Architectural Heritage Objects Based on Abkhazia and Chechnya Architectural Monuments Dating back from 2nd to 11th Centuries, 2nd International Conference on Art Studies: Science, Experience, Education (ICASSEE 2018). Advances in Social Science, Education and Humanities Research 284, 613-617 (2018). https://doi.org/10.2991/icassee-18.2018.121

5. V. Pishchulina, V. Kotlyar, A. Argun, The medieval lime mortars for carrying out dating of monuments (on the example of objects of Abkhazia of the 2-11 th c.), Topical Problems of Architecture, Civil Engineering and Environmental Economics (TPACEE 2018). E3S Web Conf. 91, 02006 (2019). https://doi.org/10.1051/e3sconf/20199102006

6. J. Sanjurjo-Sánchez, International Journal of Architectural Heritage 10, 5, 620-635 (2016). https://doi.org/10.1080/15583058.2015.1055384

7. C. Batt, Encyclopedia of Scientific Dating Methods. Springer, Dordrecht (2013). https://doi.org/10.1007/978-94-007-6326-5_11-1

8. J. Mattinson, Encyclopedia of Scientific Dating Methods. Springer, Dordrecht (2014). https://doi.org/10.1007/978-94-007-6326-5_56-3

9. K.J. Thomsen, Encyclopedia of Scientific Dating Methods. Springer, Dordrecht. (2013). https://doi.org/10.1007/978-94-007-6326-5_121-2

10. N. Aluker, M. Herrmann, J. Suzdaltseva, IOP Conf. Series: Earth and Environmental Science 539, 012032 (2020). https://doi.org/10.1088/1755-1315/539/1/012032

11. C. Goedicke, K. Slusallek, M. Kubelik, Journal of the Society of Architectural Historians 40 (3), 203-217 (1981). https://doi.org/10.2307/989694

12. R. Baydoun, O. El Samad, B. Nsouli, G. Younes, Radiocarbon 57(1), 153-159 (2015). https://doi.org/10.2458/azu_rc.57.18108 
13. F. Marra, E. D’Ambrosio, M. Gaeta, M. Mattei, Archaeometry 58(2), 177-200 (2015). https://doi.org/10.1111/arcm.12154

14. E.M. Shcherban, S.A. Stelmakh, A. Sysoev, A. Chernil'nik, MSF 1022, 71-79 (2021). https://doi.org/10.4028/www.scientific.net/msf.1022.71.

15. E.A. Shlyakhova, I.A. Serebryanaya, I.O. Egorochkina, A.A. Matrosov, A.N. Soloviev, IOP Conference Series: Materials Science and Engineering 1029, 1, 012047 (2021). https://doi.org/10.1088/1757-899X/1029/1/012047

16. S.A. Stel'makh, E.M. Shcherban', A.I. Shuiskii, A.Y. Prokopov, S.M. Madatyan, I.A. Parinov, A.V. Cherpakov, Appl. Sci. 10, 8055 (2020). https://doi.org/10.3390/app10228055

17. C. Resta, G. Chellini, A. De Falco, Buildings 10, 23 (2020). https://doi.org/10.3390/buildings10020023

18. M. Angiolilli, A. Gregori, Buildings 10, $49 \quad$ (2020). https://doi.org/10.3390/buildings10030049

19. D. Giaccone, U. Santamaria, M. Corradi, Heritage 3, 29-46 (2020). https://doi.org/10.3390/heritage3010003

20. F. Fernandez, S. Germinario, R. Basile, R. Montagno, K. Kapetanaki, K. Gobakis, D. Kolokotsa, A.M. Lagou, P. Dania, M.T. Enna, M. Mangiapane, P.-N. Maravelaki, Buildings 10, 1729 (2020). https://doi.org/10.3390/buildings10100172

21. S. Mignardi, C. De Vito, M. Botticelli, G. Favero, F. Balossi Restelli, L. Marinacci, S. Alkhasoneh, L. Medeghini, Heritage 4, 91-104 (2021). https://doi.org/10.3390/heritage4010005

22. Z. Pavlík, J. Pokorný, M. Pavlíková, L. Zemanová, M. Záleská, M. Vyšvařil, T. Žižlavský, Materials 12, 3557 (2019). https://doi.org/10.3390/ma12213557 